\title{
Paper QoS-based Joint User Selection and Scheduling for MU-MIMO WLANs
}

\author{
Dasari Srinivasa Rao ${ }^{1}$ and Victor Dhason Berlin Hency ${ }^{2}$ \\ ${ }^{1}$ Dept. of ECE, GMRIT, Rajam, Andhra Pradesh, India \\ ${ }^{2}$ School of Electronics Engineering, VIT University, Chennai, Tamil Nadu, India
}

https://doi.org/10.26636/jtit.2017.112217

\begin{abstract}
The shift in Multi-User Multiple Input Multiple Output (MU-MIMO) has gained attention due to its wide support in very high throughput Wireless Local Area Networks (WLANs) such as the 802.11ac. However, the full advantage of MU-MIMO can be utilized only with proper user selection and scheduling. Also, providing Quality of Service (QoS) support is a major challenge for these wireless networks. Generally, user scheduling is done with the acquisition of Channel State Information (CSI) from all the users. In MU-MIMO based WLANs, the number of CSI request increases with the number of users. This results in an increased CSI overhead and in degradation of the overall throughput. Most of the proposals in the literature have not addressed the contention in the CSI feedback clearly. Hence, in this paper a Joint User Selection and Scheduling (JUSS) scheme is discussed and its performance is evaluated in terms of throughput, delay, packet loss and fairness. In the performance comparison some wellknown Medium Access Control (MAC) protocols are considered. The proposed scheme not only enhances throughput, but also avoids contention during CSI feedback period.
\end{abstract}

Keywords-802.11ac, CSI, delay, MMSE, throughput.

\section{Introduction}

Downlink (DL) MU-MIMO [1] in 802.11ac enables simultaneous transmissions from Access Points (APs) to the user stations (STAs) with the aid of parallel streams. The current WLAN supports up to eight spatial streams [2] at the transmitter side and allows multiple users to communicate simultaneously. Hence, there is a large increase in data rates compared to legacy 802.11 WLANs. MU-MIMO uses beamforming (BF) to direct information towards the desired users. MU-MIMO is the key revolutionary technology for all next generation WLANs [3] and cellular networks [4]. The performance of these networks depends mainly on proper user selection and scheduling. The scheduling is usually performed with the help of Channel State Information (CSI) feedback obtained from the selected users. However, obtaining CSI from all the users incurs a large overhead, and increases linearly with the channel sounding frequency and the number of user stations. Hence, there is an extensive requirement to reduce the CSI overhead.

To fully realize the benefit of MU-MIMO and guarantee the required QoS [5], it is important to acquire updated CSI from all the users. Hence, there exists a trade-off between efficiency of the scheduler and the CSI overhead. Generally, the Access Point (AP) limits the number of users based on CSI feedback. The best user CSI and suitable channels need to be obtained before the user is scheduled. In 802.11 ac [6] user selection is performed randomly. However, selecting the best user group requires CSI feedback from all the users. In thr MU-MIMO scenario, the AP has to process multiple user requests simultaneously and has to schedule them in a parallel manner to maximize the overall throughput. Determining the possible user set is a problem, however, and it depends on the precoding method adopted at the AP. Although Zero-Forcing (ZF) receiver [1] is a simple technique that mitigates the multiuser inference, it has the disadvantage of noise enhancement. Hence, to avoid noise enhancement, the minimum mean square error beamforming (MMSE BF) can be used instead. Here, the MMSE$\mathrm{BF}$ precoder is used to maximize throughput and minimize multi-user interference.

In this paper, a joint user selection and scheduling (JUSS) strategy is proposed for MU-MIMO networks to maximize downlink capacity and improve the overall throughput of the system. JUSS follows the same mechanism as 802.11ac [6], the AP involves user stations in the in polling process to procure CSI feedback. Here, scheduling is performed in parallel with active user identification and selection. Also, the contention during the CSI feedback phase will be reduced. Specifically, JUSS provides a practical solution for user scheduling. First, the AP polls the users randomly and obtains the CSI from one of the users. By using this information, the AP prepares a channel trace and broadcasts it to the users along with the polling frame. Upon receiving this frame, users can determine their individual channel gains. Then, the users respond with individual gains in the form of user response packets. By using this information, the AP prepares an ordered subset of users for obtaining CSI reports. The first user with the chosen subset is allowed to be scheduled for the MU-MIMO transmission. This process will be repeated until the AP receives all CSI reports. In the proposed JUSS, multiple stations are allowed to transmit the response packets at the AP to identify the user set to be scheduled. To avoid contention during CSI feedback, the AP identifies the user group to be scheduled based on response packets. In addition, the proposed scheme is extended onto some well-known fair scheduling 
algorithms, such as round robin (RR) and proportional fair $(\mathrm{PF})$, to attain fairness among the users.

The major contributions of this paper are summarized as follows. First, some well-known user selection schemes like semi-orthogonal user selection (SUS), random user selection (RUS), and random beamforming are reviewed. Second, the design of the proposed joint user selection and scheduling protocol is discussed. Then, the concepts to realize the protocol are briefly explained. Lastly, the performance of JUSS is evaluated using Matlab. The simulation results show that the JUSS scheme obtains better throughput, and improved fairness over the 802.11ac MAC protocol. Also, the delay and packet loss comparison are made with the existing protocols.

The rest of the paper is organized as follows. In Section 2, the background and related work concerning the problem are presented. Section 3 gives the details about the proposed scheme. The concepts discussed include CSI feedback, scheduling mechanism, and MU-MIMO transmission cycle of the protocol. Then, Section 4 evaluates the performance of the scheme with various parameters applied. Finally, Section 5 concludes the paper.

\section{Background and Related Work}

\subsection{CSI Feedback Mechanism}

The MU-MIMO technique is widely adopted in current 802.11 WLANs [3] because of its several dominant features. It offers high data rates [2] to the users at high signalto-noise ratios. The AP is able to communicate simultaneously with multiple users providing spatial multiplexing. In addition, it offers considerable capacity gains [1] compared to the wireless communications system. The fundamental requirement for MU-MIMO based WLANs is to obtain CSI feedback before transmission. Generally, CSI acquisition [7] is performed by estimating a training sequence known by both the transmitter and the receiver In the uplink MU-MIMO system, the AP usually extracts the uplink CSI from the PHY preambles of the frames received. In the case of the downlink transmission, in turn, the acquisition of CSI cannot be performed directly.

CSI feedback schemes are classified depending on the computation of CSI at the two ends [8]. The first one involves implicit feedback, where the AP computes the CSI by estimating training sequences sent from the stations, while the other one - explicit feedback - means that stations calculate the CSI by estimating the training sequence sent from the AP, and then STAs feedback the calibrated CSI to the AP. Implicit feedback experiences less overhead compared to the explicit variety. However, in a practical wireless LAN system, channel variations and interference seen at the user stations is quite different from what is seen at the AP. Hence, the antennas at the AP are to be calibrated to eliminate the distortion if the implicit feedback scheme is used. On the other hand, the explicit feedback scheme provides higher CSI resolution at the cost of an increased overhead.

In literature, MAC control frames are usually extended to support CSI feedback, while an Explicit Compressed Feedback (ECFB) scheme [6] is implemented in IEEE 802.11ac to schedule and compress the amount of CSI feedback. The basic transmission cycle of a 802.11ac WLAN is shown in Fig. 1. It does not matter which CSI feedback scheme is applied. Both in the case of implicit and explicit schemes, the number of CSI requests in an MU-MIMO system increases with a growing number of users. This, in turn, affects the performance of the system.

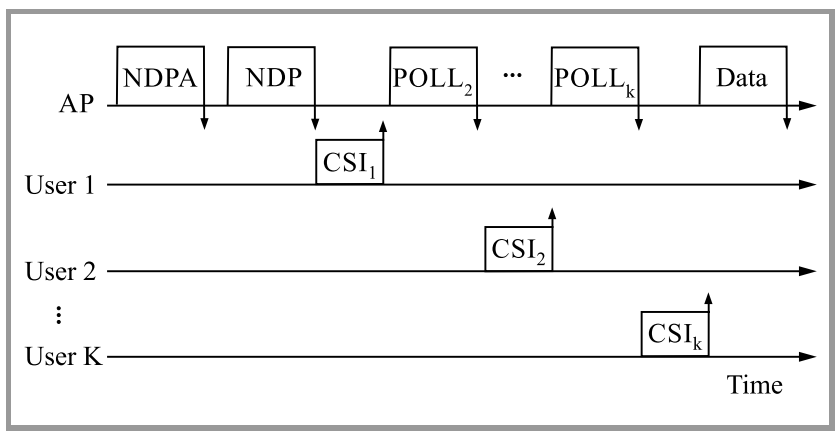

Fig. 1. 802.11ac MU-MIMO transmission cycle.

As evidenced by the discussion presented above, the major problem with CSI acquisition consists in a channel sounding interval and overhead due to feedback from one or more stations. Most publications have discussed the feedback overhead reduction using compression techniques [9]. However, compression leads to a loss of throughput in MUMIMO transmissions. The quantization of feedback information is already adopted by Long Term Evolution (LTE) and MU-MIMO based Wi-Fi systems. Though extensive work [9], [10] has been done with regard to quantizing bits, there is still some room to optimize the feedback content. In addition to compression and quantization, sending the feedback information to the AP in an adaptive manner is another alternative enabling to reduce overload rates.

One such possible solution is provided in [11], where users with signal-to-noise ratios (SNRs) above a certain threshold value need to report the CSI to the AP. However, these techniques may not result in higher channel gains, as they ignore the relation between the user subchannels. Subchannels refer to the group of sub-carriers in orthogonal frequency division multiplexing (OFDM). In [12], the author presented a subchannel access approach, where the subchannels are allocated to users with the highest SNR to maximize the transmission rate and to keep the throughput of each user as high as possible. Since the scheduler works based on the SNR measurements received from the physical layer, most of the time is spent on the identification of the best user with the largest SNR. The size of the subchannel allocation table increases with the number of users and adds complexity to the scheduler.

Another challenge faced by MU-MIMO wireless LANs involves the frequency of CSI requests. The accuracy of the 
system will be high if the feedback period is shorter than the coherence time. But, at the same time, this leads to a huge overhead at the AP. Hence, the frequency of CSI requests should be optimized. The authors in [13] and [14] have shown the impact of the channel sounding interval on the performance of the system. The key objective of the papers discussed above is to reduce the CSI feedback overhead with the increase in the number of users. It can be achieved with the help of adaptive CSI feedback mechanisms.

\subsection{User Selection/Grouping Process}

Nowadays, Wi-Fi systems are being deployed in crowded scenarios like airports, stadiums and buildings, where the number of concurrent users to be served is sufficiently large. In such situations, identifying, selecting and grouping the active users is a major task. In the MU-MIMO environment an AP with $M$ antennas may be able to select a subset of $M$ users out of all $K$ users in a network. The problem of selecting users depends on the MAC scheduler and on the information provided by the PHY layer. User selection requires CSI estimates from all user stations. But even with the availability of full CSI knowledge at the AP, it is still difficult to determine the optimal user set that maximizes the transmission rate, specifically when the number of $K$ users is large. Hence, an efficient user selection/grouping algorithm has to be devised to identify and select the user STAs to be co-scheduled.

In [15], the author discussed a Semi-orthogonal User Selection (SUS) algorithm, which achieves the maximum sumrate with low complexity. The threshold is set to satisfy orthogonality and the users with the highest channel quality are selected.

Another popular algorithm, Orthogonality Probing based User Selection (OPUS) [16] has presented a user selection algorithm that is scalable and enables the user to evaluate its orthogonality with existing users. The author also discussed the distributed contention mechanism that singles out the best user to feedback its CSI. It outperforms conventional user selection schemes in terms of throughput and fairness.

In 802.11ac each user is selected randomly [6] with equal probability and requires $M$ CSI feedbacks. Most publications have considered sounding all users before the transmission, whereas in [14] the author provided the flexibility to choose the subset of users that were sounded most recently. This exploited another direction and recently in [17] the author coupled the sounding, selecting and scheduling of users to maintain throughput and fairness performance. In 18], the author proposed a novel orthogonality evaluation mechanism that enables each user to obtain its own CSI. This algorithm is known as signpost. Signpost also realizes a $2 \mathrm{D}$ prioritized contention mechanism to choose the best user efficiently by using both time and frequency domain resources. Signpost is a scalable user selection algorithm that is suited for uplink MU-MIMO WLAN transmissions. In this protocol, for each contention round, arbitrary prob- ing directions are transmitted as channel hint to the user stations. The user stations check the orthogonality using these arbitrary directions and contend for the channel without sending the feedback to the access point. Hence, with zero CSI overhead the user competes for the resources.

Another recent uplink MU-MIMO WLAN protocol, known as optimal user selection (OUS) is proposed in [19]. OUS takes throughput and fairness into consideration and formulates the complex scheduling problem. OUS also considers the correlation among the users and provides throughput fairness solution to the user selection problem. It studies the impact of grouping the users based on throughput and fairness. To gain a better understanding of the above schemes, a summary of various state-of-art user selection and scheduling schemes with consideration of key design issues is provided in Table 1.

Table 1

Performance metrics of CSI feedback schemes

\begin{tabular}{|c|c|c|c|c|}
\hline \multirow[b]{2}{*}{$\begin{array}{l}\text { Scheme/ } \\
\text { algorithm }\end{array}$} & \multicolumn{4}{|c|}{ Design issues considered } \\
\hline & $\begin{array}{c}\text { Through- } \\
\text { put }\end{array}$ & $\begin{array}{l}\text { Fair- } \\
\text { ness }\end{array}$ & $\begin{array}{l}\text { Over- } \\
\text { head }\end{array}$ & $\begin{array}{c}\text { Com- } \\
\text { plex- } \\
\text { ity }\end{array}$ \\
\hline SUS [15] & $\checkmark$ & $\checkmark$ & High & Low \\
\hline OPUS [16] & $\checkmark$ & $\checkmark$ & Low & High \\
\hline $802.11 \mathrm{ac}+[17]$ & $\checkmark$ & $\checkmark$ & Low & High \\
\hline Signpost [18] & $\checkmark$ & $\checkmark$ & Low & High \\
\hline OUS [19] & $\checkmark$ & $\checkmark$ & - & Low \\
\hline
\end{tabular}

\subsection{User Scheduling Schemes}

When there are users contending for the same resource, their throughput may be improved by scheduling the users with fair channel quality. So, the increase in performance is obtained by exploring multi user diversity among the users. Due to this, the throughput of low rate users will be affected. System performance must be maximized without compromising fairness among the users. In user scheduling, the most challenging task is to determine the optimal user set because of the large search space. Hence, determining the optimal user set and scheduling them based on the QoS requirements improves the overall throughput.

With the introduction of MU-MIMO, most of the research regarding WLANs is now focusing on solving the issues affecting MU-MIMO based WLANs. In this way, multi user scheduling has gained attention. In [20], the author presented a novel MAC design with opportunistic MU-MIMO scheduling based on channel sounding feedback. Here, the packets being selected are based on transmission duration and the type of traffic. In [21], an efficient and heuristic MU-MIMO transmission method was proposed and compared to the beamforming based approach. The authors of [22] investigated the MU-MIMO transmission in WLAN by extending the MAC protocol with training functionalities to support efficient multi-user transmission. In [23] 
the author verified the combined effect of packet aggregation with scheduling and has shown improved throughput performance at the cost of higher delay. In [24] the author presented a distributed multi-user scheduling scheme, which enhances the total throughput under many situations, compared with both contention-less and contention-based schemes. In all the publications discussed above, fairness among the user flows is not considered. Fairness issues were studied in [25], where a multi-user proportional fair scheduling scheme was proposed to schedule multi-user transmissions while providing a high degree of fairness.

\section{JUSS Design}

\subsection{System Model}

In this section, we consider the proposed MU-MIMO system with MMSE [26] as the precoder. The AP consists of $M$ antennas and $K$-single antenna user stations that communicate with each other as shown in Fig. 2. The data symbols are transmitted through $M$ antennas, and assume the MIMO channel satisfies the Rayleigh fading conditions: $h_{k}$ are zero mean unit variance complex Gaussian channel vector. $w_{k}$ is the beamforming weight corresponding to the linear precoder. MMSE precoder eliminates multi-user interference and provides better noise immunity compared to conventional ZFBF receiver.

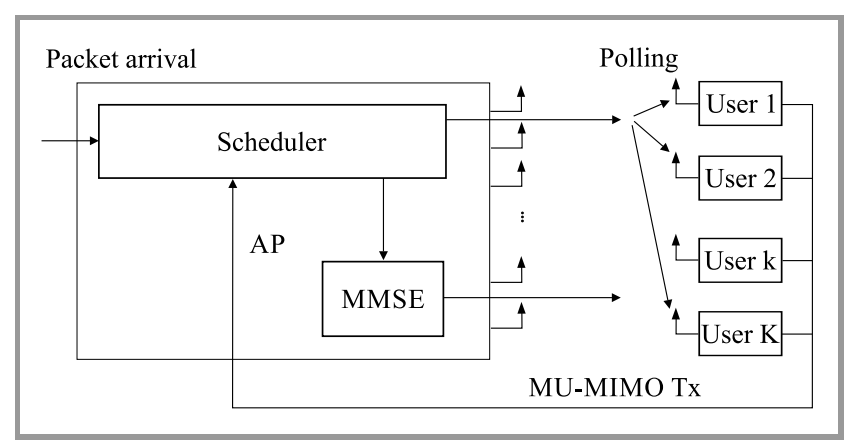

Fig. 2. MU-MIMO DL system model.

In MMSE precoding, since independent information is transmitted through different antennas the symbols are uncorrelated which means the precoding vector of one user is orthogonal to other users. Let $S$ be the group of selected users, then the precoding matrix $\mathbf{W}(S)$ is given by:

$$
\mathbf{W}(S)=P_{d}\left[P_{d} \mathbf{H}(S)^{*} \mathbf{H}(S)+\sigma_{n}^{2} \mathbf{I}\right]^{-1} \mathbf{H}(S)^{*},
$$

where $P_{d}$ represents the power of the transmitted data symbols, $\sigma_{n}^{2}$ is the noise variance, $\mathbf{H}(S)$ and $\mathbf{H}(S)^{*}$ the channel matrix, conjugate transpose matrix respectively.

\subsection{Design Overview}

In the proposed scheme, the CSI feedback and scheduling stages are considered jointly. The main idea is illustrated in Fig. 3. At the start of a transmission, the AP polls the first user (user 1 in the diagram) and it responds with its CSI. Then, the AP immediately puts user 1 in the scheduled user set $S$. User 1 is selected based on the AP queuing policy. Based on the first user CSI, the AP prepares a channel trace which is transmitted to all users. The channel trace typically contains the effective channel gain and the channel probe direction. This is termed as dual alignment metric. This information will be sent as the help of this information, the users can know contend for resources by transmitting the contention announcement (CA) frame. Then, the user with the best alignment metric will be announced as the winner in the contention round. In the example shown, user 2 is declared as the winner of that round. This step will be repeated until the AP successfully receives all $M$ CSI reports or the feedback timeout is notified. In order to gain better understanding, the step-by-step procedure for the MU-MIMO downlink transmission is explained below:

1. Consider an AP with $M$ antennas and $K$ single antenna user stations. Assume that the channel is Gaussian with zero mean and unit variance (i.e. $h_{k}$ satisfies i.i.d.).

2. The AP computes the precoding matrix $(\mathbf{W}(S)=$ $\left.\mathbf{H}(S)^{*}\right)$ the pseudo-inverse, where $S$ is the group of selected users. Now, AP starts polling the users.

3. At the beginning, AP polls the user 1 and it replies with its CSI, by making use of this information the AP prepares a channel trace.

4. Then, AP broadcasts the channel trace to the remaining users.

5. Upon receiving the channel trace from the AP, each user calculates individual gains and the user with the effective gain and the lowest correlation (i.e. better orthogonality) will be added to the set (may be user 2 in the example).

6. Repeat the steps 3-5 until the AP acquires all $M$ CSI reports.

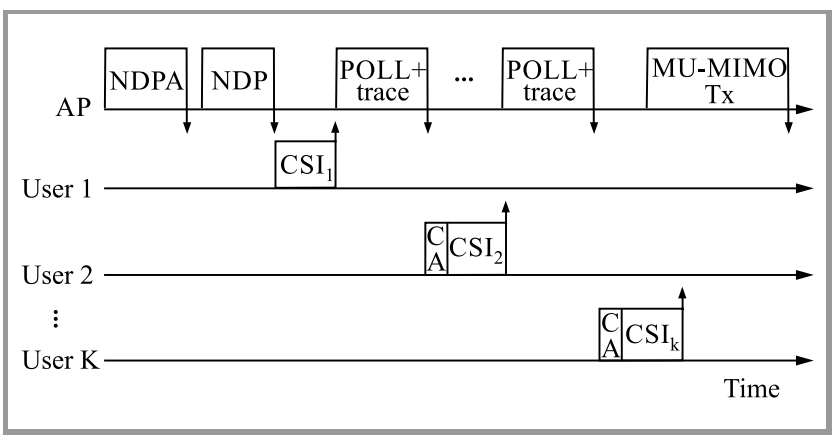

Fig. 3. MU-MIMO CSI feedback mechanism for JUSS.

The key idea behind the proposed scheme is that instead of entailing the AP to obtain the CSI from all the users, JUSS runs a distributed user selection and scheduling algorithm, where users participate in scheduling decisions to improve 
the downlink capacity. As a result, the AP undergoes limited CSI feedback exchanges.

\subsection{Feedback Mechanism}

The user scheduling in the proposed scheme utilizes the channel gain obtained from the individual user stations during the feedback stage. Based on the information obtained from the user station, the AP prepares a channel trace that is basically composed of two preference metrics. This is termed as dual alignment metric. The first one gives the channel gain magnitude of the user station, and the other metric gives the probe direction of the desired user station. We shall look into the concept of these metrics in detail. For each user $k$, compute $v_{k}$ the component of $h_{k}$ orthogonal to the subspace spanned by $\left\{v_{(1)}, v_{(2)}, \ldots, v_{(|S|)}\right\}$ :

$$
v_{k}=h_{k}-\sum_{j=1}^{|S|} \frac{h_{k} v_{(j)}^{*}}{\left\|v_{(j)}\right\|^{2}} v_{(j)}=h_{k}\left(\mathbf{I}-\sum_{j=1}^{|S|} \frac{v_{(j)}^{*} v_{(j)}}{\left\|v_{(j)}\right\|^{2}}\right) .
$$

The effective channel gain of user $k$ is denoted as $v_{k}$, it is seen that $\left\{v_{(j)}, 1 \leq j \leq|S|\right\}$ is a set of orthogonal vectors in $C^{1 \times M}, S$ is the set of users. The largest projection component is generally selected as the best user in this algorithm. Another important metric that is required to single out the best user is channel direction. After obtaining the channel state vector from the first user, the AP computes the possible directions to probe the signal space, which is orthogonal to selected users. The MU-MIMO MMSE system precodes the data symbols towards the desired users by suppressing the noise and interference. Let $M$ be the number of transmit antennas on the access point and $N$ be the number of client users. There will be a maximum of $M$ rounds to feedback CSI. Each client user computes the alignment metric between the channel state vector $h_{k}$ and the $i$-th probe vector $p_{i}$ :

$$
g_{k}=\frac{\left\|h_{k} p_{i}\right\|^{2}}{\left\|h_{k}\right\|^{2}} .
$$

The orthogonality requirement for $p_{i}$ is given by:

$$
\left.\begin{array}{l}
h_{k} \cdot p_{i}=0, k \in S \\
P=\operatorname{NullH}_{n-1}
\end{array}\right\},
$$

where $P$ is the probe vector and $n$ is the number of contending rounds. Using the above metrics, the active users contend for the channel during the MU-MIMO transmission. The key advantage of this scheme is that the next best user will be decided among the users with the help of the dual alignment metric in a distributed manner. The objective of the scheme is to select the best user set with orthogonality and minimize the contention using an efficient feedback mechanism.

\subsection{Contention Mechanism}

Although the dual alignment metric computed in the above section is meant to single out a preferred set of users, contention will take place if more than one user contends for the channel at the same time. This is called feedback contention. Hence, to avoid feedback contention and to minimize the collision, an efficient feedback contention mechanism is required. For a MU-MIMO network with $M$ antenna Access Point and $N$ client users the contention mechanism works as follows. Recall each user can locally compute the dual alignment metric such that the user with strong orthogonality is selected. To avoid the contention among the users the AP selects the users by allowing them to transmit in slot durations. So, the users contend during different contention times. The users who might think their metric is best will contend for resource at different slots by transmitting CA packet. Upon receiving the request from the users, the AP singles out the best user and puts includes them in the selected user set. If any user hears contention announcement on a time slot, it stops transmitting and waits for a random amount of time for the next slot. For each round one user will be announced as contention winner and there will be maximum of $M$ contention rounds. In JUSS, the number of contending users will be lower, because each user has to contend for the channel based on their individual alignment metric. So, each user actively participates in the scheduling process and, hence, the AP avoids the need to acquire CSI from all users. The fundamental difference between JUSS and 802.11ac is that the former uses a fixed CA duration for each CSI feedback and the AP modifies the polling frame by transmitting channel trace along with probing data. Also, to extend the fairness among the users, round robin and proportional fair scheduling algorithms are adopted in JUSS. These issues, along with throughput, delay and packet loss analysis, are discussed in the following section.

\section{Performance Evaluation}

For comparison purposes, we considered some well-known algorithms, namely 802.11ac, SUS with different $\alpha$ values [14] and the recent 802.11ac+ [16]. All simulations are performed using Matlab. The simulation parameters are shown in Table 2. The number of AP antennas is set to 4 and the number of serving users can equal up to 25 . The OFDM PHY is specified to operate in the $5.2 \mathrm{GHz}$ operating band. Each trace contains subcarrier (52 subcarriers) and transmission power ranges from 23 to $25 \mathrm{dBm}$. The total channel bandwidth is $160 \mathrm{MHz}$ (two $80 \mathrm{MHz}$ channels). The data traffic is generated in terms of packets from the AP and users. The proposed MAC is implemented by considering $M$ transmit antennas $(M=2,4)$ and $K$ user stations ( $K$ varies up to 25 ).

\subsection{Throughput Performance}

To illustrate the throughput gain of user selection/scheduling over 802.11ac, Figure 4 shows the throughput of the proposed MMSE beamforming scheme for $M=2,4$ access point antennas. It is clearly seen that throughput of the downlink system degrades as the number of polls increases. It also affects the overall throughput. 
Table 2

Simulation parameters

\begin{tabular}{|c|c|}
\hline Parameters & Values \\
\hline \hline Carrier frequency & $5.2 \mathrm{GHz}$ \\
\hline Channel bandwidth & $160 \mathrm{MHz}$ \\
\hline Number of subcarriers & 52 \\
\hline Modulation scheme & 16 QAM \\
\hline Payload length & $512-1472$ bytes \\
\hline Tx power & $23 \mathrm{dBm}$ \\
\hline MAC protocol & Extended CSMA/CA \\
\hline
\end{tabular}

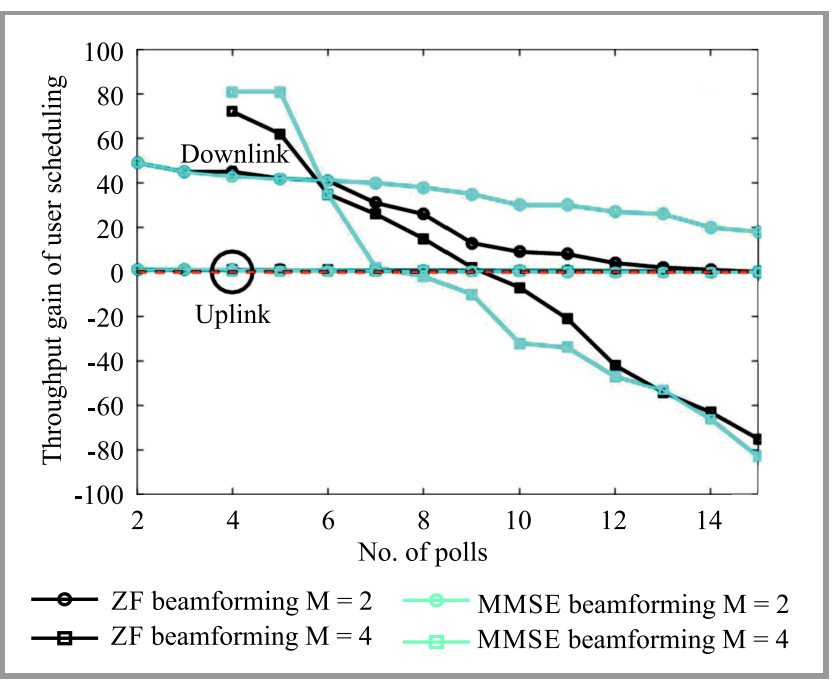

Fig. 4. Throughput gain of user selection/scheduling over 802.11ac. (See color pictures online at www.nit.eu/publications/journal-jtit)

The performance of any scheduling scheme is generally affected by an increase in the number of users. Although the increase in the number of scheduled users will benefit user diversity, the CSI feedback overhead may ultimately limit the gain. The impact of the user rate on the downlink throughput is shown in Fig. 5. The downlink and system throughputs of the three protocols are obtained. It is shown that the JUSS scheme performs well when compared to 802.11ac and SUS. It achieves a high throughput gain over $802.11 \mathrm{ac}$ and SUS respectively.

\subsection{Throughput-Fairness Performance}

The system throughput of 802.11ac+ and JUSS protocols is compared based on the round robin and proportional fairness criteria. It is shown in Fig. 6 that the system throughput of round robin and proportional fair JUSS is much higher than 802.11ac and better than RR-802.11ac+, PF-802.11ac+. JUSS and its fair approaches show similar performance, although they adopt different user selection criteria. Particularly, the system throughput of JUSS is far above that of 802.11ac. In Fig. 7. the downlink throughput attained by each user is plotted. From the plot, it is clear that the JUSS scheme achieves throughput that is higher than in 802.11ac and 802.11act.

The proposed scheme shows also that users with low SNRs achieve good throughput as well. This is possible because of MMSE precoding in the user selection process. The proposed scheme also achieves fair throughput over all users. It can be seen in the graph that 802.11ac does not show fair performance in the case of all users, hence the users with high SNRs will benefit the most. However, in the proposed scheme, the user grouping is done based on the alignment metric available at the AP.

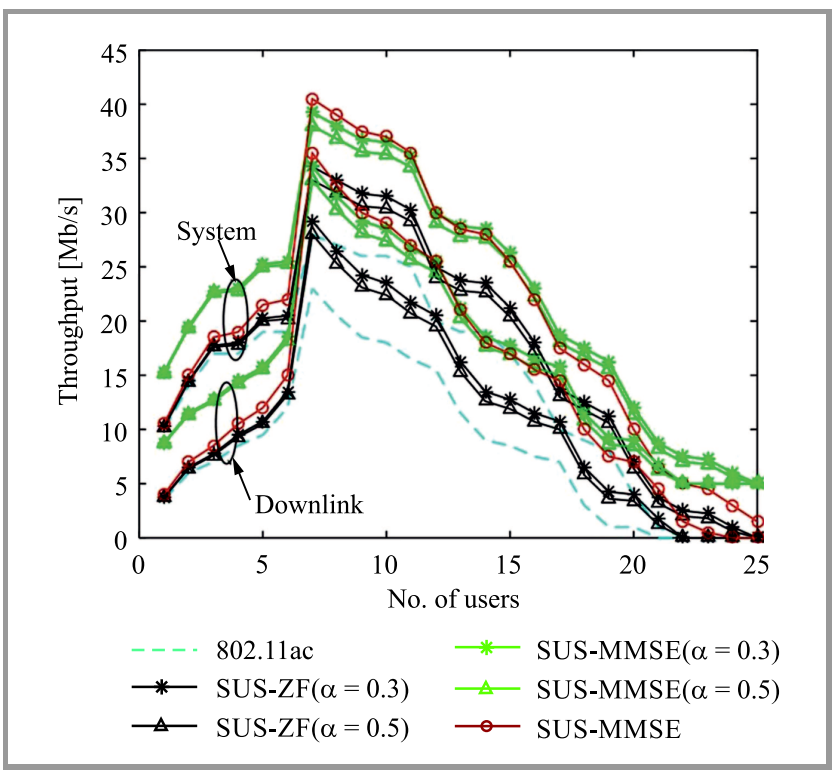

Fig. 5. System and downlink throughput with respect to the number of users.

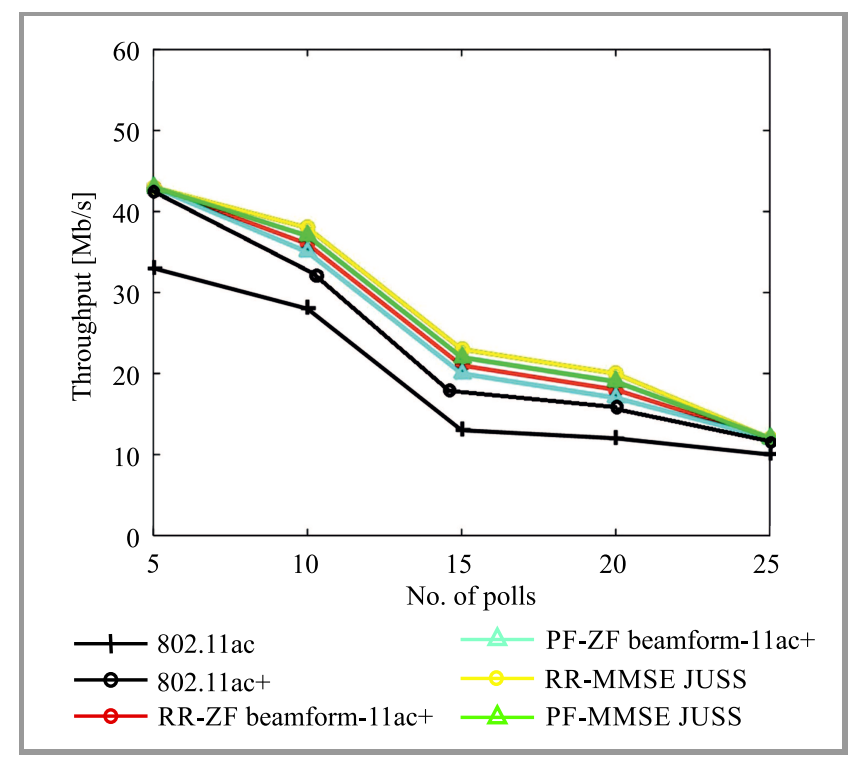

Fig. 6. System throughput comparison with respect to the number of polls. 


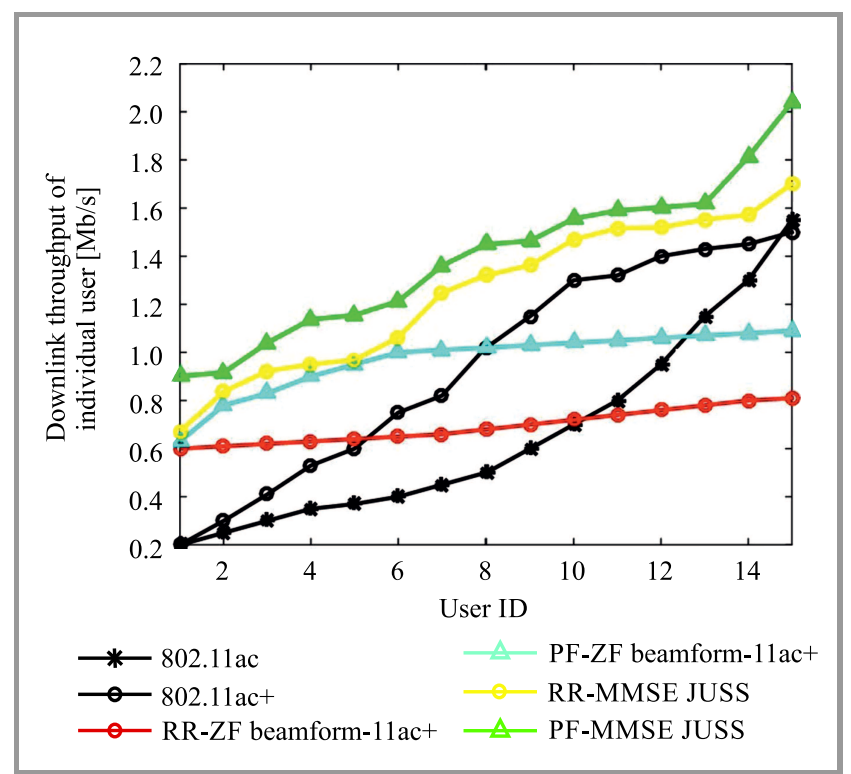

Fig. 7. Downlink throughout comparison of 802.11ac, $802.11 \mathrm{ac}+$ and JUSS.

\subsection{Delay Performance}

Figure 8 gives the packet delay with respect to the number of interfering users. It is clearly shown that the percentage of packets lost for JUSS is low compared to ZF beamforming. The proposed feedback contention mechanism reduces packet loss due to collisions. In Fig. 9, the delay performance for ZF beamforming and MMSE beamforming scheduling schemes are provided. It indicates that the proposed scheme incurs less delay compared to the $\mathrm{ZF}$ beamforming method.

Some important conclusions about the results are given below.

- at $M=4$ transmit antennas, our proposed scheduling scheme achieves better throughput gain over conventional ZF beamforming schedulers,

- the throughput comparison with 802.11ac, SUS and $802.11 \mathrm{ac}+$ shows that, JUSS outperforms the existing schemes,

- it also provides fairness among the users along with throughput performance,

- in addition, it is also shown that the proposed scheme gives less delay and reduced packet loss compared to the ZF beamforming method.

\section{Conclusions}

In this work, an MMSE based MU-MIMO DL protocol for WLANs is proposed. The proposed JUSS scheme uses a dual alignment metric to select the preferred user set and schedule the MU-MIMO transmission collectively. The main advantage of this scheme consists in the fact that it eliminates the need to collect CSI from all users and reduces the feedback overhead. MMSE not only reduces

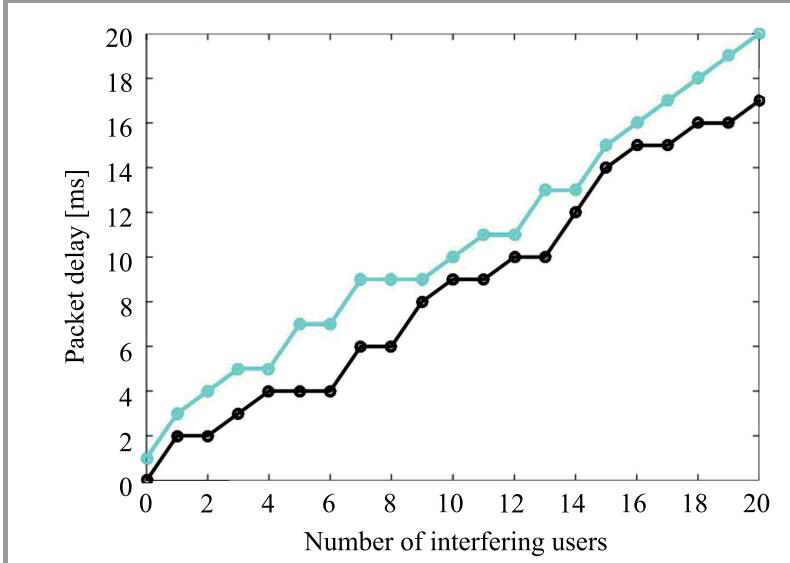

- MMSE beamforming method $\rightarrow$ ZF beamforming method

Fig. 8. Packet delay vs. number of interfering users

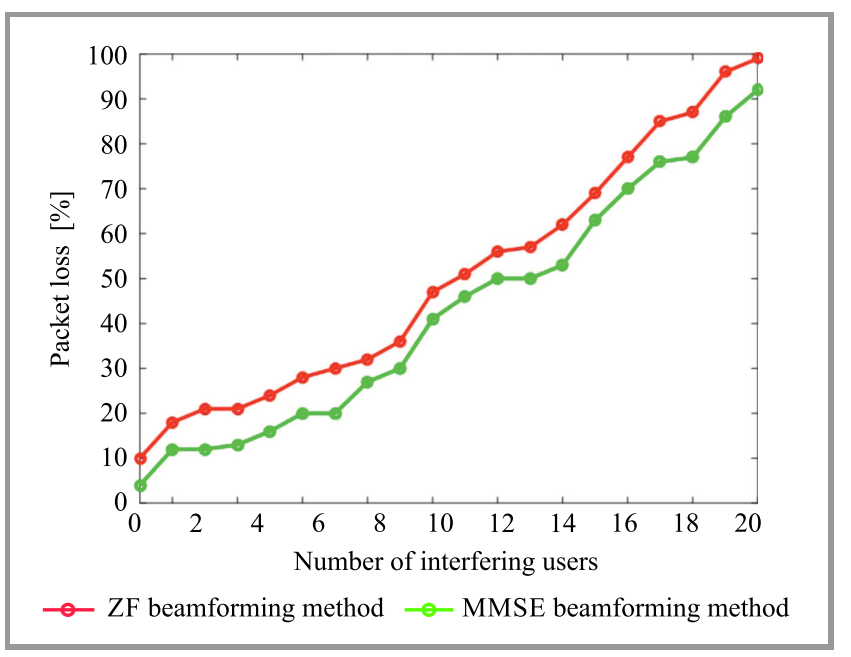

Fig. 9. Packet loss vs. number of interfering users.

interference, but also suppresses the noise present in the information. For performance comparison protocols like 802.1lac, SUS and recent 802.11ac+ are considered. To evaluate the performance of the proposed scheme throughput, fairness, delay and packet loss metrics are considered in this paper. The results obtained show that JUSS gives a better throughput performance compared to 802.11ac, SUS and 802.11ac+ protocols. Also, JUSS provides good results in terms of QoS performance measures like fairness, delay and packet loss.

\section{References}

[1] D. Tse and P. Viswanath, Fundamentals of Wireless Communication, 2 ed. Cambridge: Cambridge University Press, 2005.

[2] Cisco, "802.11ac: The Fifth Generation of Wi-Fi", in Cisco White Paper, 2012, p. 125.

[3] E. Parahia and R. Stacey, Next generation wireless LANs: 802.11n and 802.11ac, 2 ed. Cambridge: Cambridge University Press, 2013.

[4] Evolved Universal Terrestrial Radio Access (E-UTRA), Downlink Multiple Input Multiple Output (MIMO) enhancement for LTE Advanced (Release 11). 3GPP TR V11.0.0., 2015 [Online]. Available: http://www.3gpp.org 
[5] D. Srinivasa Rao and V. Berlin Hency, "QoS based Radio Resource Management Techniques for Next Generation MU-MIMO WLANs: A Survey", J. of Telecom. Electr. and Comp. Engin., vol. 8, no. 1, pp. 97-105, 2016.

[6] IEEE, IEEE Std P802.11ac: Part 11: Wireless LAN Medium Access Control (MAC) and Physical Layer (PHY) specifications: enhancements for very high throughput for operation in bands below $6 \mathrm{GHz}$, 2013.

[7] R. Liao, B. Bellalta, M. Oliver, and Z. Niu, "MU-MIMO MAC protocols for wireless local area networks: A survey", arXiv: 1404.1622v2 (doi: 10.1109/COMST.2014.2377373).

[8] H. Lou, M. Ghosh, P. Xia, and R. Olesen, "A comparison of implicit and explicit channel feedback methods for MUMIMO WLAN systems", in Proc. 2013 IEEE 24th Ann. Inter. Symp. on Person., Indoor, and Mob. Radio Commun. PIMRC, London, UK, 2013, pp. 419-424 (doi: 10.1109/PIMRC.2013.6666172).

[9] X. Xie, X. Zhang, and K. Sundaresan, "Adaptive feedback compression for MIMO networks", in Proc. of the 19th Ann. Int. Conf. on Mob. Comput. \& Network. MobiCom'13, Miami, FL, USA, 2013, pp. 453-464 (doi: 10.1145/2500423.2500465).

[10] D. Love, R. Heath, V. Lau, D. Gesbert, B. Rao, and M. Andrews, "An overview of limited feedback in wireless communication systems", IEEE J. on Selec. Areas in Commun., vol. 26, no. 8, pp. 1341-1365, 2008.

[11] V. Hassel et al., "A threshold-based channel state feedback algorithm for modern cellular systems", IEEE Trans. on Wireless Commun., vol. 6, no. 7, pp. 2422-2426, 2007.

[12] A. B. Makhlouf and M. Hamdi, "Dynamic multiuser sub-channels allocation and real-ime aggregation model for IEEE 802.11 WLANs", IEEE Trans. on Wireless Commun., vol. 13, no. 11, pp. 6015-6026 (doi: 10.1109/TWC.2014.2353611).

[13] G. Redieteab, L. Cariou, P. Christin, and J. F. Hélard, "PHY+MAC channel sounding interval analysis for IEEE 802.11ac MUMIMO", in Proc. 9th IEEE Int. Symp. on Wireless Commun. Syst. ISWCS 2012, Paris, France, 2012, pp. 1054-1058 (doi: 10.1109/ISWCS.2012.6328529).

[14] O. Bejarano, E. Magistretti, O. Gurewitz, and E. W. Knightly, "MUTE: sounding inhibition for MU-MIMO WLANs", in Proc. 11th Ann. IEEE Int. Conf. on Sensing, Commun., and Network. SECON, Singapore, Singapore, 2014, pp. 135-143 (doi: 10.1109/SAHCN.2014.6990336).

[15] T. Yoo, N. Jindal, and A. Goldsmith, "Multi-antenna downlink channels with limited feedback and user selection", IEEE J. on Selec. Areas. in Commun., vol. 25, no. 7, 2007, pp. 1478-1491 (doi: 10.1109/JSAC.2007.070920).

[16] X. Xie and X. Zhang, "Scalable user selection for MU-MIMO networks", in Proc. IEEE Int. Conf. on Comp. Commun. INFOCOM 2014, Toronto, ON, Canada, 2014, pp. 808-816 (doi: 10.1109/INFOCOM.2014.6848008)

[17] K. Lee and C. Kim, "User scheduling for MUMIMO transmission with active CSI feedback", EURASIP J. on Wireless Commun. and Network., vol. 112, 2015 (doi: 10.1186/s13638-015-0331-4).

[18] A. Zhou, T. Wei, X. Zhang, M. Liu, and Z. Li, "Signpost: Scalable MU-MIMO signaling with zero CSI feedback", in Proc. of the 16th ACM Int. Symp. on Mob. Ad Hoc Network. and Comput. MobiHoc'15, Hangzhou, Zhejiang, China, 2015, pp. 327-336 (doi: 10.1145/2746285.2746286).

[19] Y. Zhou, A. Zhou, and M. Liu, "OUS: Optimal user selection in MU-MIMO WLANs", in Proc. Int. Conf. on Comput., Network. and Comm. ICNC 2016, Kauai, HI, USA, 2016 (doi: 10.1109/ICCNC.2016.7440681).

[20] S. Wu, W. Mao, and X. Wang, "Performance study on a CSMA/CAbased MAC protocol for multi-user MIMO wireless LANs", IEEE Trans. on Wireless Commun., vol. 13, no. 6, pp. 3153-3166 (doi: 10.1109/TWC.2014.042314.131407).

[21] S. Huang, H. Yin, J. Wu, and V. C. M. Leung, "User selection for multiuser MIMO downlink with zero-forcing beamforming", IEEE Trans. on Vehic. Technol., vol. 62, no. 7, pp. 3084-3097, 2013 (doi: 10.1109/TVT.2013.2244105).
[22] M. X. Gong, E. Perahia, R. Want, and S. Mao, "Training protocols for multi-user MIMO wireless LANs, in Proc. IEEE 21st Int. Symp. on Person. Indoor and Mob. Rad. Commun. PIMRC 2010, Istanbul, Turkey, 2010, pp. 1218-1223 (doi: 10.1109/PIMRC.2010.5672041).

[23] B. Bellalta, J. Barcelo, D. Staehle, A. Vinel, and M. Oliver, "On the performance of packet aggregation in IEEE 802.11ac MU-MIMO WLANs", IEEE Commun. Lett., vol. 16, no. 10, pp. 1588-1591, 2012.

[24] S. Tang, "Distributed multiuser scheduling for improving throughput of wireless LAN", IEEE Trans. on Wireless Commun., vol. 13, no. 5 , pp. 2770-2781. 2014 (doi: 10.1109/TWC.2013.040214.130707).

[25] V. Valls and D. J. Leith, "Proportional fair MU-MIMO in 802.11 WLANs", IEEE Wireless Commun. Lett., vol. 3, no. 2, pp. 221-224, 2014 (doi: 10.1109/WCL.2014.020314.130884).

[26] V. Jones and H. Sampath, "Emerging technologies for WLAN", IEEE Commun. Mag., vol. 53, no. 3, pp. 141-49, 2015 (doi: 10.1109/MCOM.2015.7060496).

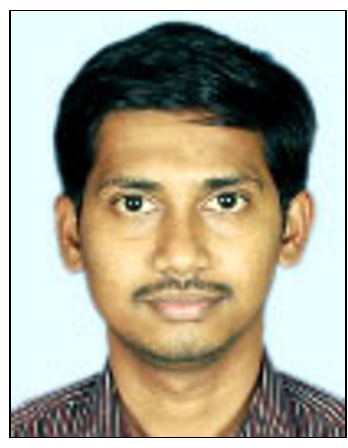

Dasari Srinivasa Rao received his Masters in Engineering (communication systems) from Thiagarajar College of Engineering, Madurai. Now, he is pursuing a Ph.D. from VIT University, Chennai. He has nearly eight years of experience in teaching. He is currently working as an Asst. Professor at Dept. of ECE, GMR Institute of Technology, Rajam, India. His research interests include wireless communications, next generation networks, and coding techniques.

E-mail: srinivasa.dasari@gmail.com

Department of ECE GMR Institute of Technology

Rajam, Andhra Pradesh, India

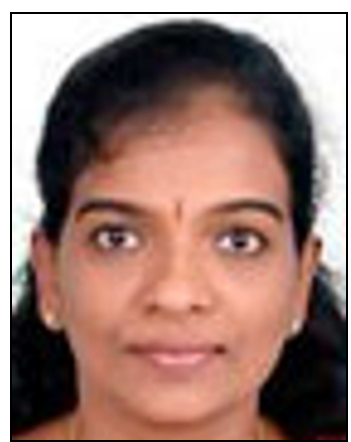

Victor Berlin Hency received a B.E. in Electronics and Communication Engineering from Manonmaniam Sundarana University. She received a M.E. in Applied Electronics from Sathyabama University. She received a Ph.D. degree in Information and Communication Engineering from Anna University. She is an Associate Professor at the school of Electronics Engineering, VIT University Chennai Campus. Her research interests include wireless networks and IoT.

E-mail: berlinhency.victor@vit.ac.in

School of Electronics Engineering

VIT University

Chennai, Tamil Nadu, India 